\title{
A class of differential hemivariational inequalities in Banach spaces
}

\author{
Stanisław Migórski ${ }^{1,2}$. Shengda Zeng ${ }^{3}$
}

Received: 30 March 2018 / Accepted: 21 May 2018 / Published online: 25 May 2018

(C) The Author(s) 2018

\begin{abstract}
In this paper we investigate an abstract system which consists of a hemivariational inequality of parabolic type combined with a nonlinear evolution equation in the framework of an evolution triple of spaces which is called a differential hemivariational inequality [(DHVI), for short]. A hybrid iterative system corresponding to (DHVI) is introduced by using a temporally semi-discrete method based on the backward Euler difference scheme, i.e., the Rothe method, and a feedback iterative technique. We apply a surjectivity result for pseudomonotone operators and properties of the Clarke subgradient operator to establish existence and a priori estimates for solutions to an approximate problem. Finally, through a limiting procedure for solutions of the hybrid iterative system, the solvability of (DHVI) is proved without imposing any convexity condition on the nonlinear function $u \mapsto f(t, x, u)$ and compactness of $C_{0}$-semigroup $e^{A(t)}$.
\end{abstract}

Keywords Differential hemivariational inequality $\cdot C_{0}$-semigroup $\cdot$ Rothe method . Pseudomonotone $\cdot$ Clarke subdifferential

Project supported by the National Science Center of Poland under Maestro Project No. UMO-2012/06/A/ST1/00262, and the National Science Center of Poland under Preludium Project No. 2017/25/N/ST1/00611. The first author is also supported by Qinzhou University Project No. 2018KYQD06, and the International Project co-financed by the Ministry of Science and Higher Education of Republic of Poland under Grant No. 3792/GGPJ/H2020/2017/0.

Shengda Zeng

shengdazeng@gmail.com; shdzeng@hotmail.com; zengshengda@163.com

Stanisław Migórski

stanislaw.migorski@uj.edu.pl

1 College of Sciences, Qinzhou University, Qinzhou 535000, Guangxi, People’s Republic of China

2 Chair of Optimization and Control, Jagiellonian University in Krakow, ul. Lojasiewicza 6, 30348 Kraków, Poland

3 Faculty of Mathematics and Computer Science, Jagiellonian University in Krakow, ul. Lojasiewicza 6, 30348 Kraków, Poland 
Mathematics Subject Classification $35 \mathrm{~L} 15 \cdot 35 \mathrm{~L} 86 \cdot 35 \mathrm{~L} 87 \cdot 74 \mathrm{Hxx} \cdot 74 \mathrm{M} 10$

\section{Introduction}

It is well known that the theory of variational inequalities, which was initially developed to deal with equilibrium problems, is closely related to the convexity of the energy functionals involved, and is based on various monotonicity arguments. If the corresponding energy functionals are nonconvex (also called superpotentials), another type of inequalities arises as variational formulation of a problem. They are called hemivariational inequalities and their derivation is based on properties of the Clarke subgradient defined for locally Lipschitz functions. Note that in contrast to variational inequalities, the stationary hemivariational inequalities are not equivalent to minimization problems, they produce substationarity problems, which study started with the pioneering works of Panagiotopoulos, see [37,38] and the references therein. Since in our life, many problems are described by nonsmooth superpotentials, it is not surprising that, during the last thirty years, a lot of scholars devoted their work to the development of theory and applications of hemivariational inequalities, for example, in contact mechanics [14,35,36,44,51], well-posedness [28,49], control problems [31], nonconvex and nonsmooth inclusions $[42,43]$, and so forth.

Furthermore, the notion of differential hemivariational inequalities was firstly introduced by Liu et al. [27] in 2016. Interest in differential hemivariational inequalities originated, similarly as in differential variational inequalities. Differential variational inequalities (DVIs) were firstly systematically discussed by Pang and Stewart [41] in Euclidean spaces, because (DVIs) are useful to represent models involving both dynamics and constraints in the form of inequalities which arise in many applied problems, for example, mechanical impact problems, electrical circuits with ideal diodes, the Coulomb friction problems for contacting bodies, economical dynamics, dynamic traffic networks, and so on. After the work [41], more and more scholars are attracted to boost the development of theory and applications for (DVIs). For instance, Liu et al. [22] in 2013 studied the existence and global bifurcation problems for periodic solutions to a class of differential variational inequalities in finite dimensional spaces by using the topological methods from the theory of multivalued maps and some versions of the method of guiding functions, Gwinner [12] in 2013 obtained a stability result of a new class of differential variational inequalities by using the monotonicity method and the technique of the Mosco convergence, and Chen and Wang [8] in 2014 used the idea of (DVIs) to investigate a dynamic Nash equilibrium problem of multiple players with shared constraints and dynamic decision processes. For more details on this topics in finite dimensional spaces the reader is welcome to consult $[7,12,13,18,19,29,30,39,40,46-48,53]$ and the references therein.

It is noteworthy that all aforementioned works were considered only in finite dimensional spaces. But, in our life, many applied problems in engineering, operations research, economical dynamics, and physical sciences, etc., are more precisely described by partial differential equations. Based on this motivation, recently, Liu-Zeng-Motreanu [24,26] in 2016 and Liu et al. [23] in 2017 proved the existence of solutions for a class of differential mixed variational inequalities in Banach spaces through applying the theory of semigroups, the Filippov implicit function lemma and fixed point theorems for condensing set-valued operators. However, until now, only one reference, Liu et al. [27], considered a differential hemivariational inequality in Banach spaces which is constituted by a nonlinear evolution equation and a hemivariational inequality of elliptic type rather than of parabolic type. Also, in the paper [27], the authors required that the constraint set $K$ is bounded, the nonlinear 
function $u \mapsto f(t, x, u)$ maps convex subsets of $K$ to convex sets and the $C_{0}$-semigroup $e^{A(t)}$ is compact. Therefore, in our present work, we would like to overcome those flaws, fill a gap, and develop new mathematical tools and methods for differential hemivariational inequalities.

Let $V, E, X$ and $Y$ be reflexive, separable Banach spaces, $H$ be a separable Hilbert space, $A: D(A) \subset E \rightarrow E$ be the infinitesimal generator of $C_{0}$-semigroup $e^{A t}$ in $E$ and

$$
\begin{aligned}
& f:(0, T) \times E \times Y \rightarrow E, \\
& \vartheta: H \rightarrow Y, \\
& \mathscr{N}: V \rightarrow V^{*}, \\
& M: V \rightarrow X, \\
& J: E \times X \rightarrow \mathbb{R}, \\
& F:(0, T) \times E \rightarrow V^{*}
\end{aligned}
$$

be given maps, which will be specified in the sequel. In this paper, we consider the following abstract system consisting of a hemivariational inequality of parabolic type combined with a nonlinear abstract evolution equation.

Problem 1 Find $u:(0, T) \rightarrow V$ and $x:(0, T) \rightarrow$ E such that

$$
\begin{aligned}
& x^{\prime}(t)=A x(t)+f(t, x(t), \vartheta u(t)) \text { for a.e. } t \in(0, T) \\
& \left(u^{\prime}(t), v\right)_{H}+\langle\mathscr{N}(u(t)), v\rangle+J^{0}(x(t), M u(t) ; M v) \geq\langle F(t, x(t)), v\rangle \\
& \quad \text { for all } v \in V \text { and a.e. } t \in(0, T) \\
& x(0)=x_{0} \text { and } u(0)=u_{0} .
\end{aligned}
$$

The main novelties of the paper are described as follows. First, for the first time, we apply the Rothe method, see [16,51], to study a system of a hemivariational inequality of parabolic type driven by a nonlinear abstract evolution equation. Until now, there are a few papers devoted to the Rothe method for hemivariational inequalities, see [4,5,52]. Furthermore, all of them investigated only a single hemivariational inequality by using Rothe method.

Second, the main results can be applied to a special case of Problem 1 in which the locally Lipschitz functional $J$ and the nonlinear function $F$ are assumed to be independent of the variable $x$. So, Problem 1 reduces to the following hemivariational inequality of parabolic type: find $u:(0, T) \rightarrow V$ such that $u(0)=u_{0}$ and

$$
\left(u^{\prime}(t), v\right)_{H}+\langle\mathscr{N}(u(t)), v\rangle+J^{0}(M u(t) ; M v) \geq\langle F(t), v\rangle
$$

for all $v \in V$ and a.e. $t \in(0, T)$. This problem was considered only recently by MigórskiOchal [33], Kalita [17], and Fang et al. [11].

Third, until now, all contributions concerning (DVIs) were driven only by variational/hemivariational inequalities of elliptic type. Here, for the first time, we discuss (DHVI) governed by a hemivariational inequality of parabolic type. Additionally, in comparison with our previous works $[23,24,26,27]$, in this paper, we do not impose any convexity assumption on the nonlinear function $u \mapsto f(t, x, u)$ and we remove the compactness hypothesis on $C_{0}$-semigroup $e^{A(t)}$.

The paper is organized as follows. In Sect. 2, we recall some definitions and preliminary facts concerning nonlinear and nonsmooth analysis, which will be used in the sequel. In Sect. 3, we provide the definition of a solution to Problem 1 in the mild sense, and then establish a hybrid iterative system, Problem 16. The solvability of Problem 16 is obtained by a surjectivity result for a pseudomonotone operator and a priori estimate for the solutions to 
Problem 16 is proved. Finally, through a limiting procedure for the solutions to Problem 16, the existence of solution to Problem 1 is established.

\section{Preliminaries}

This section is devoted to recall basic notation, definitions and some auxiliary results from nonlinear analysis, see $[9,10,36,50]$, which will be used in the sequel.

We start with definitions and properties of semicontinuous set-valued mappings.

Definition 2 Let $X$ and $Y$ be topological spaces, and $F: X \rightarrow 2^{Y}$ be a set-valued mapping. We say that $F$ is

(i) upper semicontinuous (u.s.c., for short) at $x \in X$ if, for every open set $O \subset Y$ with $F(x) \subset O$ there exists a neighborhood $N(x)$ of $x$ such that

$$
F(N(x)):=\cup_{y \in N(x)} F(y) \subset O .
$$

If this holds for every $x \in X$, then $F$ is called upper semicontinuous.

(ii) lower semicontinuous (l.s.c., for short) at $x \in X$ if, for every open set $O \subset Y$ with $F(x) \cap O \neq \emptyset$ there exists a neighborhood $N(x)$ of $x$ such that

$$
F(y) \cap O \neq \emptyset \text { for all } y \in N(x) .
$$

If this holds for every $x \in X$, then $F$ is called lower semicontinuous.

(iii) continuous at $x \in X$ if, it is both upper semicontinuous and lower semicontinuous at $x \in X$. If this holds for every $x \in X$, then $F$ is called continuous.

The following theorem gives some criteria for the upper semicontinuity of set-valued mappings.

Proposition 3 (see [36]) Let $X, Y$ be two topological spaces and $F: X \rightarrow 2^{Y}$. The following statements are equivalent

(i) $F$ is u.s.c.,

(ii) for every closed set $C \subset Y$, the set $F^{-}(C):=\{x \in X \mid F(x) \cap C \neq \emptyset\}$ is closed in $X$,

(iii) for every open set $O \subset Y$, the set $F^{+}(O):=\{x \in X \mid F(x) \subset O\}$ is open in $X$.

Next, we recall the definition of pseudomonotonicity of a single-valued operator.

Definition 4 Let $X$ be a reflexive Banach space with dual $X^{*}$ and $A: X \rightarrow X^{*}$. We say that $A$ is pseudomonotone, if $A$ is bounded and for every sequence $\left\{x_{n}\right\} \subseteq X$ converging weakly to $x \in X$ such that $\lim \sup \left\langle A x_{n}, x_{n}-x\right\rangle \leq 0$, we have

$$
\langle A x, x-y\rangle \leq \liminf _{n \rightarrow \infty}\left\langle A x_{n}, x_{n}-y\right\rangle \text { for all } y \in X .
$$

Remark 5 It is known that an operator $A: X \rightarrow X^{*}$ is pseudomonotone, if and only if $x_{n} \rightarrow x$ weakly in $X$ and $\limsup _{n \rightarrow \infty}\left\langle A x_{n}, x_{n}-x\right\rangle \leq 0$ entails

$$
\lim _{n \rightarrow \infty}\left\langle A x_{n}, x_{n}-x\right\rangle=0 \text { and } A x_{n} \rightarrow A x \text { weakly in } X^{*} .
$$

Furthermore, if $A \in \mathcal{L}\left(X, X^{*}\right)$ is nonnegative, then it is pseudomonotone.

Next, the pseudomonotonicity of multivalued operators is defined below. 
Definition 6 A multivalued operator $T: X \rightarrow 2^{X^{*}}$ is pseudomonotone if

(a) for every $v \in X$, the set $T v \subset X^{*}$ is nonempty, closed and convex,

(b) $T$ is upper semicontinuous from each finite dimensional subspace of $X$ to $X^{*}$ endowed with the weak topology,

(c) for any sequences $\left\{u_{n}\right\} \subset X$ and $\left\{u_{n}^{*}\right\} \subset X^{*}$ such that $u_{n} \rightarrow u$ weakly in $X, u_{n}^{*} \in T u_{n}$ for all $n \geq 1$ and $\lim \sup \left\langle u_{n}^{*}, u_{n}-u\right\rangle \leq 0$, we have that for every $v \in X$, there exists $u^{*}(v) \in T u$ such that

$$
\left\langle u^{*}(v), u-v\right\rangle \leq \liminf _{n \rightarrow \infty}\left\langle u_{n}^{*}, u_{n}-v\right\rangle .
$$

Definition 7 Given a locally Lipschitz function $J: X \rightarrow \mathbb{R}$ on a Banach space $X$, we denote by $J^{0}(u ; v)$ the generalized (Clarke) directional derivative of $J$ at the point $u \in X$ in the direction $v \in X$ defined by

$$
J^{0}(u ; v)=\limsup _{\lambda \rightarrow 0^{+}, w \rightarrow u} \frac{J(w+\lambda v)-J(w)}{\lambda} .
$$

The generalized gradient of $J: X \rightarrow \mathbb{R}$ at $u \in X$ is defined by

$$
\partial J(u)=\left\{\xi \in X^{*} \mid J^{0}(u ; v) \geq\langle\xi, v\rangle \text { for all } v \in X\right\} .
$$

The following result provides an example of a multivalued pseudomonotone operator which is a superposition of the Clarke subgradient with a compact operator. The proof can be found in [3, Proposition 5.6].

Proposition 8 Let $V$ and $X$ be two reflexive Banach spaces, $\gamma: V \rightarrow X$ be a linear, continuous, and compact operator. We denote by $\gamma^{*}: X^{*} \rightarrow V^{*}$ the adjoint operator to $\gamma$. Let $j: X \rightarrow \mathbb{R}$ be a locally Lipschitz functional such that

$$
\|\partial j(v)\|_{X^{*}} \leq c_{j}\left(1+\|v\|_{X}\right) \text { for all } v \in V
$$

with $c_{j}>0$. Then the multivalued operator $G: V \rightarrow 2^{V^{*}}$ defined by

$$
G(v)=\gamma^{*} \partial j(\gamma(v)) \text { for all } v \in V
$$

is pseudomonotone.

Moreover, we recall the following surjectivity result, which can be found in [10, Theorem 1.3.70] or [50].

Theorem 9 Let $X$ be a reflexive Banach space and $T: X \rightarrow 2^{X^{*}}$ be pseudomonotone and coercive. Then $T$ is surjective, i.e., for every $f \in X^{*}$, there exists $u \in X$ such that $T u \ni f$.

We now introduce spaces of functions, defined on a finite interval $[0, T]$. Let $\pi$ denote a finite partition of the interval $(0, T)$ by a family of disjoint subintervals $\sigma_{i}=\left(a_{i}, b_{i}\right)$ such that $[0, T]=\cup_{i=1}^{n} \bar{\sigma}_{i}$. Let $\mathcal{F}$ denote the family of all such partitions. For a Banach space $X$ and $1 \leq q<\infty$, we define the space

$$
B V^{q}(0, T ; X)=\left\{v:[0, T] \rightarrow X \quad \mid \sup _{\pi \in \mathcal{F}}\left\{\sum_{\sigma_{i} \in \pi}\left\|v\left(b_{i}\right)-v\left(a_{i}\right)\right\|_{X}^{q}\right\}<\infty\right\}
$$

and define the seminorm of a vector function $v:[0, T] \rightarrow X$ by

$$
\|v\|_{B V^{q}(0, T ; X)}^{q}=\sup _{\pi \in \mathcal{F}}\left\{\sum_{\sigma_{i} \in \pi}\left\|v\left(b_{i}\right)-v\left(a_{i}\right)\right\|_{X}^{q}\right\} .
$$


Assume that $1 \leq p \leq \infty$ and $1 \leq q<\infty$, and $X, Z$ are Banach spaces such that $X \subset Z$ with continuous embedding. We introduce the following Banach space

$$
M^{p, q}(0, T ; X, Z)=L^{p}(0, T ; X) \cap B V^{q}(0, T ; Z),
$$

which is endowed with the norm $\|\cdot\|_{L^{p}(0, T ; X)}+\|\cdot\|_{B V^{q}(0, T ; Z)}$. Recall a useful compactness result, which proof can be found in [17, Proposition 2.8].

Proposition 10 Let $1 \leq p, q<\infty$, and $X_{1} \subset X_{2} \subset X_{3}$ be Banach spaces such that $X_{1}$ is reflexive, the embedding $X_{1} \subset X_{2}$ is compact, and the embedding $X_{2} \subset X_{3}$ is continuous. If a set $B$ is bounded in $M^{p, q}\left(0, T ; X_{1}, X_{3}\right)$, then $B$ is relatively compact in $L^{p}\left(0, T ; X_{2}\right)$.

We end this section by recalling a discrete version of the Gronwall inequality, which can be found in [15, Lemma 7.25] and [45, Lemma 2.32].

Lemma 11 Let $T>0$ be given. For a positive integer $N$, we define $\tau=\frac{T}{N}$. Assume that $\left\{g_{n}\right\}_{n=1}^{N}$ and $\left\{e_{n}\right\}_{n=1}^{N}$ are two sequences of nonnegative numbers satisfying

$$
e_{n} \leq \bar{c} g_{n}+\bar{c} \tau \sum_{j=1}^{n-1} e_{j} \text { for } n=1, \ldots, N
$$

for a positive constant $\bar{c}$ independent of $N$ (or $\tau)$. Then there exists a positive constant $c$, independent of $N$ (or $\tau$ ), such that

$$
e_{n} \leq c\left(g_{n}+\tau \sum_{j=1}^{n-1} g_{j}\right) \text { for } n=1, \ldots, N .
$$

\section{Main results}

In this section, we focus our attention on the investigation of an abstract system, which consists of a hemivariational inequality of parabolic type, and a nonlinear evolution equation involving an abstract semigroup operator. The method of proof is based on properties of subgradient operators in the sense of Clarke, surjectivity of multivalued pseudomonotone operators, the Rothe method, and convergence analysis.

We begin this section with the standard notation and function spaces, which can be found in $[9,10,50]$. Let $(V,\|\cdot\|)$ be a reflexive and separable Banach space with its dual space $V^{*}$, $H$ be a separable Hilbert space, and $\left(Y,\|\cdot\|_{Y}\right)$ be another reflexive and separable Banach space. Subsequently, we assume that the spaces $V \subset H \subset V^{*}$ (or $\left(V, H, V^{*}\right)$ ) form an evolution triple of spaces (see cf. [36, Definition 1.52]) with dense, continuous, and compact embeddings. The embedding injection from $V$ to $H$ is denoted by $\iota: V \rightarrow H$. Moreover, let $\left(X,\|\cdot\|_{X}\right)$ and $\left(E,\|\cdot\|_{E}\right)$ be reflexive and separable Banach spaces with their duals $X^{*}$ and $E^{*}$, respectively. For $0<T<+\infty$, in the sequel, we use the standard Bochner-Lebesgue function spaces $\mathcal{V}=L^{2}(0, T ; V), \mathcal{H}=L^{2}(0, T ; H), \mathcal{X}=L^{2}(0, T ; X), \mathcal{V}^{*}=L^{2}\left(0, T ; V^{*}\right)$ and $\mathcal{W}=\left\{v \in \mathcal{V} \mid v^{\prime} \in \mathcal{V}^{*}\right\}$, here $v^{\prime}$ denotes the time derivative of $v$, understood in the sense of distributions. The notation $\langle\cdot, \cdot\rangle_{\mathcal{V}^{*} \times \mathcal{V}}$ stands for the duality between $\mathcal{V}$ and $\mathcal{V}^{*}$. The space of linear bounded operaors from $V$ to $X$ is denoted by $\mathcal{L}(V, X)$.

To prove the solvability of Problem 1, we impose the following assumptions on the data of the problem.

$H(A): A: D(A) \subset E \rightarrow E$ is the infinitesimal generator of a $C_{0}$-semigroup $e^{A t}$ in $E$.

$\overline{H(\mathscr{N})}: \mathscr{N}: V \rightarrow V^{*}$ is a pseudomonotone operator such that 
(i) $\langle\mathscr{N} v, v\rangle \geq a_{0}\|v\|^{2}-a_{1}\|v\|_{H}^{2}$ for all $v \in V$.

(ii) one of the following conditions holds

(ii) ${ }_{1} \mathscr{N}$ satisfies the growth condition

$$
\|\mathscr{N}(v)\|_{V^{*}} \leq a_{2}+a_{3}\|v\|
$$

for all $v \in V$ with $a_{2} \geq 0, a_{3}>0$.

(ii) $2 \widetilde{\mathscr{N}}$ is bounded in $\mathcal{V} \cap L^{\infty}(0, T ; H)$ and

$$
\widetilde{N}\left(u_{n}\right) \rightarrow \widetilde{\mathscr{N}}(u) \text { weakly in } \mathcal{V}^{*}
$$

for any sequence $\left\{u_{n}\right\}$ with $u_{n} \rightarrow u$ weakly in $\mathcal{V}$, where $\widetilde{\mathscr{N}}: \mathcal{V} \rightarrow \mathcal{V}^{*}$ is the Nemytskii operator for $\mathscr{N}$ defined by $(\widetilde{\mathscr{N}} u)(t)=\mathscr{N}(u(t))$ for $t \in[0, T]$.

$\underline{H(J)}: J: E \times X \rightarrow \mathbb{R}$ is a functional such that

(i) $u \mapsto J(x, u)$ is locally Lipschitz for all $x \in E$.

(ii) there exists $c_{J}>0$ such that

$$
\|\partial J(x, u)\|_{X^{*}} \leq c_{J}\left(1+\|u\|_{X}\right) \text { for all } u \in X \text { and } x \in E .
$$

(iii) $(x, u) \mapsto J^{0}(x, u ; v)$ is upper semicontinuous from $E \times X$ into $\mathbb{R}$ for all $v \in X$.

$H(M): M \in \mathcal{L}(V, X)$ and its Nemytskii operator $\mathcal{M}: M^{2,2}\left(0, T ; V, V^{*}\right) \rightarrow \mathcal{X}$ defined by $\overline{(\mathcal{M u}(t))}=M u(t)$ for $t \in[0, T]$ is compact.

$H(F): F:(0, T) \times E \rightarrow V^{*}$ is an operator such that

(i) $t \mapsto F(t, x)$ is measurable for all $x \in E$.

(ii) $x \mapsto F(t, x)$ continuous for all $t \in[0, T]$.

(iii) there exists a constant $m_{F}>0$ such that $\|F(t, x)\|_{V^{*}} \leq m_{F}$ for all $(t, x) \in(0, T) \times E$.

$H(0): a_{0}>c_{J}\|M\|^{2}$.

$\overline{H(\vartheta)}: \vartheta: H \rightarrow Y$ is a compact operator.

$\overline{H(f)}: f:(0, T) \times E \times Y \rightarrow E$ is such that

(i) $t \mapsto f(t, x, u)$ is measurable for every $(x, u) \in E \times Y$.

(ii) $(x, u) \mapsto f(t, x, u)$ is continuous for a.e. $t \in(0, T)$.

(iii) there exists a positive function $\varphi \in L^{2}(0, T)$ such that

$$
\left\{\begin{array}{l}
\left\|f\left(t, x_{1}, u\right)-f\left(t, x_{2}, u\right)\right\|_{E} \leq \varphi(t)\left\|x_{1}-x_{2}\right\|_{E}, \\
\|f(t, 0, u)\|_{E} \leq \varphi(t)\left(1+\|u\|_{Y}\right)
\end{array}\right.
$$

for a.e. $t \in(0, T)$, all $x_{1}, x_{2} \in E$ and $u \in Y$.

Remark 12 We provide two examples of operator $\mathscr{N}$ which satisfies the hypotheses $H(\mathscr{N})$. In the first example, assume that $V=H_{0}^{1}(\Omega)$ and $\mathscr{N}: V \rightarrow V^{*}$ is a second order quasilinear differential operator in divergence form of the Leray-Lions type, i.e.,

$$
\langle\mathscr{N} u, v\rangle=\sum_{i=1}^{d} \int_{\Omega} a_{i}(x, \nabla u(x)) D_{i} v(x) d x
$$

for all $u, v \in V$, where $\Omega$ is an open bounded subset of $\mathbb{R}^{d}, d=2,3, D_{i}=\frac{\partial}{\partial x_{i}}, \nabla=$ $\left(D_{1}, D_{2}, \ldots, D_{d}\right)$, and each $a_{i}$ is a Carathéodory function such that

$\left(A_{1}\right)$ there exist $c_{1}>0$ and $b_{1} \in L^{2}(\Omega)$ such that

$$
\left|a_{i}(x, \xi)\right| \leq c_{1}\|\xi\|+b_{1}(x) \text { for a.e. } x \in \Omega \text { and all } \xi \in \mathbb{R}^{d} .
$$


$\left(A_{2}\right) \sum_{i=1}^{d}\left(a_{i}\left(x, \xi_{1}\right)-a_{i}\left(x, \xi_{2}\right)\right) \cdot\left(\xi_{1}-\xi_{2}\right) \geq 0$ for a.e. $x \in \Omega$, all $\xi_{1}, \xi_{2} \in \mathbb{R}^{d}$.

$\left(A_{3}\right)$ there exist a constant $c_{2}>0$ and a nonnegative function $b_{2} \in L^{1}(\Omega)$ such that

$$
\sum_{i=1}^{d} a_{i}(x, \xi) \xi_{i} \geq c_{2} \sum_{i=1}^{d}\left|\xi_{i}\right|^{2}-b_{2}(x)
$$

for a.e. $x \in \Omega$ and all $\xi \in \mathbb{R}^{d}$.

Then, it is well known, see [21], that $\mathscr{N}$ satisfies conditions $H(\mathscr{N})(i)$ and $(i i)_{1}$.

In the second example, $\mathscr{N}$ is an abstract Navier-Stokes operator, see [32,33]. Let $\Omega$ be a simply connected domain in $\mathbb{R}^{d}, d=2,3$ with regular boudary $\Gamma$, and

$$
W=\left\{w \in C^{\infty}\left(\Omega ; \mathbb{R}^{d}\right) \mid \operatorname{div} w=0 \text { in } \Omega, w_{T}=0 \text { on } \Gamma\right\},
$$

where $w_{T}$ is the tangential component of $w$ on the boundary $\Gamma$. Also, let $V$ and $H$ be the closure of $W$ in the norm of $H^{1}\left(\Omega ; \mathbb{R}^{d}\right)$ and $L^{2}\left(\Omega ; \mathbb{R}^{d}\right)$, respectively. Let $\mathscr{N}: V \rightarrow V^{*}$ be the classical Navier-Stokes operator, i.e., $\mathscr{N}(v)=\mathscr{N}_{1}(v)+\mathscr{N}_{2}[v]$ for all $v \in V$, where $\mathscr{N}_{1}: V \rightarrow V^{*}$ and $\mathscr{N}_{2}[\cdot]: V \rightarrow V^{*}$ are defined by

$$
\begin{aligned}
& \left\langle\mathscr{N}_{1} u, v\right\rangle=v \int_{\Omega} \operatorname{curl} u \cdot \operatorname{curl} v d x, \\
& \left\langle\mathscr{N}_{2}(u, v), w\right\rangle=\int_{\Omega}(\operatorname{curl} u \times v) \cdot w d s, \quad \mathscr{N}_{2}[v]=\mathscr{N}_{2}(v, v)
\end{aligned}
$$

for all $u, v, w \in V$, where operator curlu stands the rotation of $u$ and $v>0$. Recall that $\Omega$ is a simply connected domain, therefore, we can see that the bilinear form

$$
(u, v)_{V}=\left\langle\mathscr{N}_{1} u, v\right\rangle=v \int_{\Omega} \operatorname{curl} u \cdot \operatorname{curl} v d x
$$

generates a norm in $V,\|u\|_{V}=(u, v)_{V}^{\frac{1}{2}}$, which is equivalent to the $H^{1}\left(\Omega ; \mathbb{R}^{d}\right)$-norm. This together with the fact

$$
\left\langle\mathscr{N}_{2}(u, v), v\right\rangle=0
$$

implies that $H(\mathscr{N})(i)$ holds. From [32, Lemma 9], we can see that the Navier-Stokes operator $\mathscr{N}$ is pseudomonotone. Hypothesis $H(\mathscr{N})(i i)_{2}$ can be obtained readily by using the same argument as in [33, Theorem 1, p.739] and [1, Theorem 1].

Next, we show that hypothesis $H(J)$ implies that the subgradient operator $\partial J$ of $J$ is upper semicontinuous in suitable topologies.

Lemma 13 Assume that $H(J)$ holds. Then the subgradient operator

$$
(E, X) \ni(y, x) \mapsto \partial J(y, x) \subset X^{*}
$$

is upper semicontinuous from $E \times X$ endowed with the norm topology to the subsets of $X^{*}$ endowed with the weak topology.

Proof From Proposition 3, it remains to verify that for any weakly closed subset $D$ of $X^{*}$, the weak inverse image $(\partial J)^{-1}(D)$ of $\partial J$ under $D$ is closed in the norm topology, where

$$
(\partial J)^{-1}(D)=\{(y, x) \in E \times X \mid \partial J(y, x) \cap D \neq \emptyset\} .
$$

Let $\left\{\left(y_{n}, x_{n}\right)\right\} \subset(\partial J)^{-1}(D)$ be such that $\left(y_{n}, x_{n}\right) \rightarrow(y, x)$ in $E \times X$, as $n \rightarrow \infty$ and $\left\{\xi_{n}\right\} \subset X^{*}$ be such that $\xi_{n} \in \partial J\left(y_{n}, x_{n}\right) \cap D$ for each $n \in \mathbb{N}$. Hypothesis $H(J)$ (ii) implies 
that the sequence $\left\{\xi_{n}\right\}$ is bounded in $X^{*}$. Hence, by the reflexivity of $X^{*}$, without loss of generality, we may assume that $\xi_{n} \rightarrow \xi$ weakly in $X^{*}$. The weak closedness of $D$ guarantees that $\xi \in D$. On the other hand, $\xi_{n} \in \partial J\left(y_{n}, x_{n}\right)$ entails

$$
\left\langle\xi_{n}, z\right\rangle_{X^{*} \times X} \leq J^{0}\left(y_{n}, x_{n} ; z\right) \text { for all } z \in X .
$$

Taking into account the upper semicontinuity of $(y, x) \mapsto J^{0}(y, x ; z)$ for all $z \in X$ and passing to the limit, we have

$$
\langle\xi, z\rangle_{X^{*} \times X}=\limsup _{n \rightarrow \infty}\left\langle\xi_{n}, z\right\rangle_{X^{*} \times X} \leq \limsup _{n \rightarrow \infty} J^{0}\left(y_{n}, x_{n} ; z\right) \leq J^{0}(y, x ; z)
$$

for all $z \in X$. Hence $\xi \in \partial J(y, x)$, and consequently, we obtain $\xi \in \partial J(y, x) \cap D$, i.e., $(y, x) \in(\partial J)^{-1}(D)$. This completes the proof of the lemma.

Now, we observe that Problem 1 can be rewritten in the following equivalent form.

Problem 14 Find $u:(0, T) \rightarrow V$ and $x:(0, T) \rightarrow$ E such that

$$
\begin{aligned}
& x^{\prime}(t)=A x(t)+f(t, x(t), \vartheta u(t)) \text { for a.e. } t \in(0, T) \\
& u^{\prime}(t)+\mathscr{N}(u(t))+M^{*} \partial J(x(t), M u(t)) \ni F(t, x(t)) \text { for a.e. } t \in(0, T) \\
& x(0)=x_{0} \text { and } u(0)=u_{0} .
\end{aligned}
$$

According to our previous work $[23,25,26]$, we give the following definition of a solution to Problem 14 in the mild sense.

Definition 15 A triple of functions $(x, u, \xi)$ with $x \in C(0, T ; E), u \in \mathcal{W}$ and $\xi \in \mathcal{X}^{*}$ is said to be a mild solution of Problem 14, if

$$
\begin{aligned}
& x(t)=e^{A t} x_{0}+\int_{0}^{t} e^{A(t-s)} f(s, x(s), \vartheta u(s)) d s \text { for a.e. } t \in(0, T) \\
& u^{\prime}(t)+\mathscr{N}(u(t))+M^{*} \xi(t)=F(t, x(t)) \text { for a.e. } t \in(0, T) \\
& u(0)=u_{0},
\end{aligned}
$$

where $\xi(t) \in \partial J(x(t), M u(t))$ for a.e. $t \in(0, T)$.

In what follows, we establish the existence of a mild solution to Problem 14. We use the idea of the Rothe method combined with a feedback iterative approach.

Let $N \in \mathbb{N}, \tau=\frac{T}{N}$, and $t_{k}=k \tau$ for $k=0,1, \ldots, N$. We consider the following hybrid iterative system.

Problem 16 Find $\left\{u_{\tau}^{k}\right\}_{k=0}^{N} \subset V, x_{\tau} \in C(0, T ; E)$ and $\left\{\xi_{\tau}^{k}\right\}_{k=1}^{N} \subset X^{*}$ such that $u_{\tau}^{0}=u_{0}$ and

$$
\begin{aligned}
& x_{\tau}(t)=e^{A t} x_{0}+\int_{0}^{t} e^{A(t-s)} f\left(s, x_{\tau}(s), \vartheta \widehat{u}_{\tau}(s)\right) d s \quad \text { for a.e. } t \in\left(0, t_{k}\right) \\
& \frac{u_{\tau}^{k}-u_{\tau}^{k-1}}{\tau}+\mathscr{N}\left(u_{\tau}^{k}\right)+M^{*} \xi_{\tau}^{k}=F_{\tau}^{k} \\
& \xi_{\tau}^{k} \in \partial J\left(x_{\tau}\left(t_{k}\right), M u_{\tau}^{k}\right)
\end{aligned}
$$

for $k=1, \ldots, N$, where $F_{\tau}^{k}$ and $\widehat{u}_{\tau}(t)$ for $t \in\left(0, t_{k}\right)$ are defined by

$$
\begin{aligned}
& F_{\tau}^{k}:=\frac{1}{\tau} \int_{t_{k-1}}^{t_{k}} F\left(s, x_{\tau}(s)\right) d s \\
& \widehat{u}_{\tau}(t)= \begin{cases}u_{\tau}^{k-1}+\frac{t-t_{k}}{\tau}\left(u_{\tau}^{k-1}-u_{\tau}^{k-2}\right) & \text { for } t \in\left(t_{k-1}, t_{k}\right], 2 \leq k \leq N, \\
u_{0}, & \text { for } t \in\left[0, t_{1}\right] .\end{cases}
\end{aligned}
$$


Obviously, this system is constituted with a stationary nonlinear Clarke subdifferential inclusion and a nonlinear abstract integral equation.

First, we give the following existence result on a solution to hybrid iterative system, Problem 16.

Lemma 17 Assume that $H(A), H(F), H(\mathscr{N}), H(J), H(M), H(\vartheta), H(0)$ and $H(f)$ hold. Then, there exists $\tau_{0}>0$ such that for all $\tau \in\left(0, \tau_{0}\right)$, the hybrid iterative system, Problem 16 , has at least one solution.

Proof Given elements $u_{\tau}^{0}, u_{\tau}^{1}, \ldots, u_{\tau}^{k-1}$, it follows from definition of $\widehat{u}_{\tau}$, see (13), that $\widehat{u}_{\tau}$ is well-defined and $\widehat{u}_{\tau} \in C\left(0, t_{k} ; V\right)$. Consider the function $\mathcal{F}_{\tau}:(0, T) \times E \rightarrow E$ defined by

$$
\mathcal{F}_{\tau}(t, x)=f\left(t, x, \vartheta \widehat{u}_{\tau}(t)\right) \text { for a.e. } t \in(0, T) \text { and } x \in E .
$$

Recall that $t \mapsto f(t, x, u)$ is measurable on $(0, T)$ for all $(x, u) \in E \times Y,(x, u) \mapsto f(t, x, u)$ is continuous for a.e. $t \in(0, T)$, and $\widehat{u}_{\tau} \in C\left(0, t_{k} ; V\right)$, so we have

$$
t \mapsto \mathcal{F}_{\tau}(t, x) \text { is measurable on }(0, T) \text { for all } x \in E .
$$

From hypothesis $H(f)($ iii), we can see that $\mathcal{F}$ satisfies the following properties

$$
\left\{\begin{array}{l}
\left\|\mathcal{F}_{\tau}(t, 0)\right\|_{E} \leq \varphi(t)\left(1+\left\|\vartheta \widehat{u}_{\tau}(t)\right\|_{Y}\right) \text { for a.e. } t \in\left(0, t_{k}\right) \\
\left\|\mathcal{F}_{\tau}\left(t, x_{1}\right)-\mathcal{F}_{\tau}\left(t, x_{2}\right)\right\|_{E} \leq \varphi(t)\left\|x_{1}-x_{2}\right\|_{E} \text { for a.e. } t \in\left(0, t_{k}\right) .
\end{array}\right.
$$

These properties together with [20, Proposition 5.3, p.66] and [26, Section 4] imply that there exists a unique function $x_{\tau} \in C\left(0, t_{k} ; E\right)$ such that

$$
x_{\tau}(t)=e^{A t} x_{0}+\int_{0}^{t} e^{A(t-s)} f\left(s, x_{\tau}(s), \vartheta \widehat{u}_{\tau}(s)\right) d s \quad \text { for a.e. } t \in\left(0, t_{k}\right) .
$$

Further, from hypothesis $H(F)$ and $x_{\tau} \in C\left(0, t_{k} ; E\right)$ we can easily check

$$
F_{\tau}^{k}=\frac{1}{\tau} \int_{t_{k-1}}^{t_{k}} F\left(s, x_{\tau}(s)\right) d s \in V^{*} .
$$

It remains to find elements $u_{\tau}^{k} \in V$ and $\xi_{\tau}^{k} \in \partial J\left(x_{\tau}\left(t_{k}\right), M u_{\tau}^{k}\right)$ such that

$$
\frac{u_{\tau}^{k}-u_{\tau}^{k-1}}{\tau}+\mathscr{N}\left(u_{\tau}^{k}\right)+M^{*} \xi_{\tau}^{k}=F_{\tau}^{k}
$$

To this end, we will apply the surjective result, Theorem 9, to show that the operator $S: V \rightarrow$ $2^{V^{*}}$ defined below is onto

$$
S v=\frac{\iota^{*} \iota v}{\tau}+\mathscr{N}(v)+M^{*} \partial J\left(x_{\tau}\left(t_{k}\right), M v\right) \text { for all } v \in V .
$$

From hypothesis $H(J)($ ii), we have the following estimate

$$
\begin{gathered}
\langle\xi, M v\rangle_{X^{*} \times X} \leq\|\xi\|_{X^{*}}\|M v\|_{X} \leq c_{J}\left(1+\|M v\|_{X}\right)\|M v\|_{X} \\
\leq c_{J}\|M\|^{2}\|v\|^{2}+c_{J}\|M\|\|v\|
\end{gathered}
$$

for all $v \in V$ and $\xi \in \partial J\left(x_{\tau}\left(t_{k}\right), M v\right)$. Moreover, hypothesis $H(\mathscr{N})(\mathrm{i})$ reveals

$$
\begin{aligned}
& \langle S v, v\rangle=\frac{1}{\tau}(v, v)_{H}+\langle\mathscr{N}(v), v\rangle+\left\langle\partial J\left(x_{\tau}\left(t_{k}\right), M v\right), M v\right\rangle_{X^{*} \times X} \\
& \geq \frac{1}{\tau}\|v\|_{H}^{2}+a_{0}\|v\|^{2}-a_{1}\|v\|_{H}^{2}-\sup _{\xi \in \partial J\left(x_{\tau}\left(t_{k}\right), M v\right)}\langle\xi, M v\rangle_{X^{*} \times X} .
\end{aligned}
$$


After inserting (14) into the above inequality, we have

$$
\langle S v, v\rangle \geq\left(\frac{1}{\tau}-a_{1}\right)\|v\|_{H}^{2}+\left(a_{0}-c_{J}\|M\|^{2}\right)\|v\|^{2}-c_{J}\|M\|\|v\|
$$

for all $v \in V$. Choosing $\tau_{0}=\frac{1}{a_{1}}$ and taking into account the smallness condition $H(0)$, we conclude that $S$ is coercive for all $\tau \in\left(0, \tau_{0}\right)$. Moreover, we shall also verify that $S$ is pseudomonotone. In fact, from [36, Proposition 3.59], we know that if all components of $S$ are pseudomonotone, then $S$ is pseudomonotone as well. Since $v \mapsto \frac{\iota^{*} \iota v}{\tau}$ is bounded, linear and nonnegative, so it is pseudomonotone. On the other hand, hypotheses $H(M), H(J)(\mathrm{i})$, $H(J)$ (ii) and Proposition 8 ensure that the operator

$$
v \mapsto M^{*} \partial J\left(x_{\tau}\left(t_{k}\right), M v\right) \text { is pseudomonotone too. }
$$

Since $\mathscr{N}$ is pseudomonotone, see $H(\mathscr{N})$, we conclude by [36, Proposition 3.59] that $S$ is a pseudomonotone operator.

Consequently, by Theorem 9, we infer that there exist $u_{\tau}^{k} \in V$ and $\xi_{\tau}^{k} \in X^{*}$ such that $\xi_{\tau}^{k} \in \partial J\left(x_{\tau}\left(t_{k}\right), M u_{\tau}^{k}\right)$ and (12) holds, for all $\tau \in\left(0, \tau_{0}\right)$. This completes the proof of the lemma.

Next, we provide a result on a priori estimate for solutions to Problem 16.

Lemma 18 Assume that $H(A), H(F), H(\mathscr{N}), H(J), H(M), H(\vartheta), H(0)$ and $H(f)$ hold. Then, there exist $\tau_{0}>0$ and $C>0$ independent of $\tau$ such that for all $\tau \in\left(0, \tau_{0}\right)$, the solutions to the hybrid iterative system, Problem 16, satisfy

$$
\begin{aligned}
& \max _{1 \leq k \leq N}\left\|u_{\tau}^{k}\right\|_{H} \leq C, \\
& \sum_{k=1}^{N}\left\|u_{\tau}^{k}-u_{\tau}^{k-1}\right\|_{H} \leq C, \\
& \tau \sum_{k=1}^{N}\left\|u_{\tau}^{k}\right\|^{2} \leq C .
\end{aligned}
$$

Proof Let $\xi_{\tau}^{k} \in \partial J\left(x_{\tau}\left(t_{k}\right), M u_{\tau}^{k}\right)$ be such that equality (12) holds. Multiplying (12) by $u_{\tau}^{k}$, we have

$$
\left(\frac{u_{\tau}^{k}-u_{\tau}^{k-1}}{\tau}, u_{\tau}^{k}\right)_{H}+\left\langle\mathscr{N}\left(u_{\tau}^{k}\right), u_{\tau}^{k}\right\rangle+\left\langle\xi_{\tau}^{k}, M u_{\tau}^{k}\right\rangle_{X^{*} \times X}=\left\langle F_{\tau}^{k}, u_{\tau}^{k}\right\rangle .
$$

From $H(\mathscr{N})(\mathrm{i})$, we have

$$
\left\langle\mathscr{N}\left(u_{\tau}^{k}\right), u_{\tau}^{k}\right\rangle \geq a_{0}\left\|u_{\tau}^{k}\right\|^{2}-a_{1}\left\|u_{\tau}^{k}\right\|_{H}^{2} .
$$

Moreover, hypothesis $H(J)($ ii) guarantees that

$$
\begin{aligned}
\left\langle\xi_{\tau}^{k}, M u_{\tau}^{k}\right\rangle_{X^{*} \times X} & \geq-\left\|\xi_{\tau}^{k}\right\|_{X^{*}}\left\|M u_{\tau}^{k}\right\|_{X} \geq-c_{J}\|M\|\left(1+\left\|M u_{\tau}^{k}\right\|_{X}\right)\left\|u_{\tau}^{k}\right\| \\
& \geq-c_{J}\|M\|^{2}\left\|u_{\tau}^{k}\right\|^{2}-c_{J}\|M\|\left\|u_{\tau}^{k}\right\| .
\end{aligned}
$$

Inserting (19) and (20) into (18), and taking into account the identity

$$
(v-w, v)_{H}=\frac{1}{2}\left(\|v\|_{H}^{2}+\|v-w\|_{H}^{2}-\|w\|_{H}^{2}\right) \text { for all } v, w \in H,
$$


we obtain

$$
\begin{aligned}
\left\|F_{\tau}^{k}\right\|_{V^{*}}\left\|u_{\tau}^{k}\right\| \geq\left\langle F_{\tau}^{k}, u_{\tau}^{k}\right\rangle= & \left(\frac{u_{\tau}^{k}-u_{\tau}^{k-1}}{\tau}, u_{\tau}^{k}\right)_{H}+\left\langle\mathscr{N}\left(u_{\tau}^{k}\right), u_{\tau}^{k}\right\rangle+\left\langle\xi_{\tau}^{k}, M u_{\tau}^{k}\right\rangle_{X^{*} \times X} \\
\geq & \frac{1}{2 \tau}\left(\left\|u_{\tau}^{k}\right\|_{H}^{2}+\left\|u_{\tau}^{k}-u_{\tau}^{k-1}\right\|_{H}^{2}-\left\|u_{\tau}^{k-1}\right\|_{H}^{2}\right) \\
& +a_{0}\left\|u_{\tau}^{k}\right\|^{2}-a_{1}\left\|u_{\tau}^{k}\right\|_{H}^{2} \\
& \quad-c_{J}\|M\|^{2}\left\|u_{\tau}^{k}\right\|^{2}-c_{J}\|M\|\left\|u_{\tau}^{k}\right\| .
\end{aligned}
$$

We are now in a position to apply Cauchy's inequality with $\varepsilon>0$ to get

$$
\begin{gathered}
\varepsilon\left\|u_{\tau}^{k}\right\|^{2}+\frac{1}{4 \varepsilon}\left\|F_{\tau}^{k}\right\|_{V^{*}}^{2} \geq \frac{1}{2 \tau}\left(\left\|u_{\tau}^{k}\right\|_{H}^{2}+\left\|u_{\tau}^{k}-u_{\tau}^{k-1}\right\|_{H}^{2}-\left\|u_{\tau}^{k-1}\right\|_{H}^{2}\right) \\
+a_{0}\left\|u_{\tau}^{k}\right\|^{2}-a_{1}\left\|u_{\tau}^{k}\right\|_{H}^{2}-c_{J}\|M\|^{2}\left\|u_{\tau}^{k}\right\|^{2}-\frac{c_{J}^{2}\|M\|^{2}}{4 \varepsilon}-\varepsilon\left\|u_{\tau}^{k}\right\|^{2},
\end{gathered}
$$

that is,

$$
\begin{aligned}
& \frac{\tau}{2 \varepsilon}\left\|F_{\tau}^{k}\right\|_{V^{*}}+\frac{c_{J}^{2}\|M\|^{2} \tau}{2 \varepsilon}+2 \tau a_{1}\left\|u_{\tau}^{k}\right\|_{H}^{2} \geq\left\|u_{\tau}^{k}\right\|_{H}^{2}+\left\|u_{\tau}^{k}-u_{\tau}^{k-1}\right\|_{H}^{2} \\
& \quad-\left\|u_{\tau}^{k-1}\right\|_{H}^{2}+2 \tau\left(a_{0}-c_{J}\|M\|^{2}-2 \varepsilon\right)\left\|u_{\tau}^{k}\right\|^{2} .
\end{aligned}
$$

Summing up the above inequalities from 1 to $n$ with $1 \leq n \leq N$, we have

$$
\begin{aligned}
& 2 \tau\left(a_{0}-c_{J}\|M\|^{2}-2 \varepsilon\right) \sum_{k=1}^{n}\left\|u_{\tau}^{k}\right\|^{2}+\sum_{k=1}^{n}\left\|u_{\tau}^{k}-u_{\tau}^{k-1}\right\|_{H}^{2}+\left\|u_{\tau}^{n}\right\|_{H}^{2}-\left\|u_{\tau}^{0}\right\|_{H}^{2} \\
& \quad \leq \frac{\tau}{2 \varepsilon} \sum_{k=1}^{n}\left\|F_{\tau}^{k}\right\|_{V^{*}}+\frac{c_{J}^{2}\|M\|^{2} T}{2 \varepsilon}+2 \tau a_{1} \sum_{k=1}^{n}\left\|u_{\tau}^{k}\right\|_{H}^{2} .
\end{aligned}
$$

It follows from hypothesis $H(F)$ that $\left\|F_{\tau}^{k}\right\|_{V^{*}} \leq m_{F}$ for all $k=1,2, \ldots, N$. From the smallness condition $a_{0}>c_{J}\|M\|^{2}$, choosing $\varepsilon=\frac{a_{0}-c_{J}\|M\|^{2}}{4}$, we obtain

$$
\begin{aligned}
& \tau\left(a_{0}-c_{J}\|M\|^{2}\right) \sum_{k=1}^{n}\left\|u_{\tau}^{k}\right\|^{2}+\sum_{k=1}^{n}\left\|u_{\tau}^{k}-u_{\tau}^{k-1}\right\|_{H}^{2}+\left\|u_{\tau}^{n}\right\|_{H}^{2} \leq \frac{2 T m_{F}}{a_{0}-c_{J}\|M\|^{2}} \\
& \quad+\left\|u_{\tau}^{0}\right\|_{H}^{2}+\frac{2 c_{J}^{2}\|M\|^{2} T}{a_{0}-c_{J}\|M\|^{2}}+2 \tau a_{1} \sum_{k=1}^{n}\left\|u_{\tau}^{k}\right\|_{H}^{2} .
\end{aligned}
$$

We now apply the discrete Gronwall inequality, Lemma 11, to verify the estimates (15)-(17), which completes the proof of the lemma.

Subsequently, for a given $\tau>0$, we define the piecewise affine function $u_{\tau}$ and the piecewise constant interpolant functions $\bar{u}_{\tau}, \xi_{\tau}, \mathscr{F}_{\tau}$ as follows

$$
\begin{aligned}
& u_{\tau}(t)=u_{\tau}^{k}+\frac{t-t_{k}}{\tau}\left(u_{\tau}^{k}-u_{\tau}^{k-1}\right) \quad \text { for } t \in\left(t_{k-1}, t_{k}\right], \\
& \xi_{\tau}(t)=\xi_{\tau}^{k} \text { for } t \in\left(t_{k-1}, t_{k}\right], \\
& \bar{u}_{\tau}(t)= \begin{cases}u_{\tau}^{k}, t \in\left(t_{k-1}, t_{k}\right], \\
u_{0}, t=0,\end{cases} \\
& \mathscr{F}_{\tau}(t)=F_{\tau}^{k} \text { for } t \in\left(t_{k-1}, t_{k}\right] .
\end{aligned}
$$


For functions $u_{\tau}, \bar{u}_{\tau}$ and $\xi_{\tau}$, we have the following estimates.

Lemma 19 Assume that $H(A), H(F), H(\mathscr{N}), H(J), H(M), H(\vartheta), H(0)$ and $H(f)$ hold. Then, there exist $\tau_{0}>0$ and $C>0$ independent of $\tau$ such that for all $\tau \in\left(0, \tau_{0}\right)$, the functions $u_{\tau}, \bar{u}_{\tau}$, and $\xi_{\tau}$ satisfy

$$
\begin{aligned}
& \left\|u_{\tau}\right\|_{C(0, T ; H)} \leq C, \\
& \left\|\bar{u}_{\tau}\right\|_{L^{\infty}(0, T ; H)} \leq C, \\
& \left\|\bar{u}_{\tau}\right\|_{\mathcal{V}} \leq C, \\
& \left\|u_{\tau}\right\|_{\mathcal{V}} \leq C, \\
& \left\|\xi_{\tau}\right\|_{\mathcal{X}^{*}} \leq C, \\
& \left\|u_{\tau}^{\prime}\right\|_{\mathcal{V}^{*}} \leq C, \\
& \left\|\bar{u}_{\tau}\right\|_{M^{2,2}\left(0, T ; V, V^{*}\right)} \leq C .
\end{aligned}
$$

Proof From the estimate (15), we have

$$
\begin{aligned}
\left\|u_{\tau}(t)\right\|_{H} & \leq\left\|u_{\tau}^{k}\right\|_{H}+\frac{\left|t-t_{k}\right|}{\tau}\left\|u_{\tau}^{k}-u_{\tau}^{k-1}\right\|_{H} \\
& \leq 2\left\|u_{\tau}^{k}\right\|+\left\|u_{\tau}^{k-1}\right\| \leq C
\end{aligned}
$$

for all $t \in\left(t_{k-1}, t_{k}\right], k=1,2, \ldots, N$, hence estimate (21) holds. Also, inequality (22) is verified directly by using the estimate (15).

Moreover, the bound in (17) ensures that

$$
\begin{aligned}
& \left\|\bar{u}_{\tau}\right\|_{\mathcal{V}}^{2}=\int_{0}^{T}\left\|\bar{u}_{\tau}(t)\right\|^{2} d t=\tau \sum_{k=1}^{n}\left\|u_{\tau}^{k}\right\|^{2} \leq C, \\
& \left\|u_{\tau}\right\|_{\mathcal{V}}^{2}=\int_{0}^{T}\left\|u_{\tau}(t)\right\|^{2} d t=\sum_{k=1}^{N} \int_{t_{k-1}}^{t_{k}}\left\|u_{\tau}^{k}+\frac{\left(t-t_{k}\right)}{\tau}\left(u_{\tau}^{k}-u_{\tau}^{k-1}\right)\right\|^{2} d t \\
& \leq 10 \tau \sum_{k=1}^{N}\left\|u_{\tau}^{k}\right\|^{2} \leq C,
\end{aligned}
$$

hence, (23) and (24) are obtained. On the other hand, the hypothesis $H(J)(\mathrm{ii})$ and bound in (17) imply

$$
\begin{aligned}
\left\|\xi_{\tau}\right\|_{\mathcal{X}^{*}}^{2} & =\int_{0}^{T}\left\|\xi_{\tau}(t)\right\|_{X^{*}}^{2} d t \leq \tau \sum_{k=1}^{N}\left\|\xi_{\tau}^{k}\right\|_{X^{*}}^{2} \leq \tau \sum_{k=1}^{N} c_{J}^{2}\left(1+\left\|M u_{\tau}^{k}\right\|_{X}\right)^{2} \\
& \leq \tau \sum_{k=1}^{N} 2 c_{J}^{2}\left(1+\|M\|^{2}\left\|u_{\tau}^{k}\right\|^{2}\right) \leq 2 c_{J}^{2} T+2 c_{J}^{2}\|M\|^{2} \tau \sum_{k=1}^{N}\left\|u_{\tau}^{k}\right\|^{2} \leq C,
\end{aligned}
$$

so, $(25)$ is also verified.

Obviously, the equality (12) can be rewritten as

$$
u_{\tau}^{\prime}(t)+\mathscr{N}\left(\bar{u}_{\tau}(t)\right)+M^{*} \xi_{\tau}(t)=\mathscr{F}_{\tau}(t)
$$

for a.e. $t \in(0, T)$. Let $v \in \mathcal{V}$. We now multiply the above equality by $v$ to get

$$
\left\langle\mathscr{F}_{\tau}, v\right\rangle_{\mathcal{V}^{*} \times \mathcal{V}}-\left\langle\widetilde{\mathscr{N}}\left(\bar{u}_{\tau}\right), v\right\rangle_{\mathcal{V}^{*} \times \mathcal{V}}-\left\langle\xi_{\tau}, \mathcal{M} v\right\rangle_{\mathcal{X}^{*} \times \mathcal{X}}=\left(u_{\tau}^{\prime}, v\right)_{\mathcal{H}}=\left\langle u_{\tau}^{\prime}, v\right\rangle_{\mathcal{V}^{*} \times \mathcal{V}}
$$


Hence, we have

$$
\left\|u_{\tau}^{\prime}\right\| \mathcal{V}^{*} \leq\left\|\mathscr{F}_{\tau}\right\| \mathcal{V}^{*}+\left\|\widetilde{\mathscr{N}}\left(\bar{u}_{\tau}\right)\right\| \mathcal{V}^{*}+\|M\|\left\|\xi_{\tau}\right\|_{\mathcal{X}^{*}} .
$$

Recall that $\widetilde{N}$ is bounded in $\mathcal{V} \cap L^{\infty}(0, T ; H)$, this hypothesis combined with bounds on $\left\{\bar{u}_{\tau}\right\}$ in $\mathcal{V} \cap L^{\infty}(0, T ; H)$, see (22) and (23), implies that $\left\|\widetilde{N}\left(\bar{u}_{\tau}\right)\right\| \mathcal{V}^{*} \leq m_{0}$ for all $\tau>0$ with $m_{0}>0$ independent of $\tau$. This together with (28), estimates (23), (25), and hypothesis $H(F)$ implies that estimate (26) is satisfied.

It remains to verify the boundedness of $\left\{\bar{u}_{\tau}\right\}$ in $M^{2,2}\left(0, T ; V, V^{*}\right)$. However, from (23), we only prove that $\left\{\bar{u}_{\tau}\right\}$ is bounded in $B V^{2}\left(0, T ; V^{*}\right)$. To this end, we consider a division $0=b_{0}<b_{1}<\ldots<b_{n}=T$ with $b_{i} \in\left(\left(m_{i}-1\right) \tau, m_{i} \tau\right]$. Hence $\bar{u}_{\tau}\left(b_{i}\right)=u_{\tau}^{m_{i}}$ with $m_{0}=0, m_{n}=N$ and $m_{i+1}>m_{i}$ for $i=1,2, \ldots, N-1$. Hence, we have

$$
\begin{aligned}
\left\|\bar{u}_{\tau}\right\|_{B V^{2}\left(0, T ; V^{*}\right)}^{2} & =\sum_{i=1}^{n}\left\|u_{\tau}^{m_{i}}-u_{\tau}^{m_{i-1}}\right\|_{V^{*}}^{2} \leq \sum_{i=1}^{n}\left(m_{i}-m_{i-1}\right) \sum_{l=m_{i-1}+1}^{m_{i}}\left\|u_{\tau}^{l}-u_{\tau}^{l-1}\right\|_{V^{*}}^{2} \\
& \leq \sum_{i=1}^{n}\left(m_{i}-m_{i-1}\right) \sum_{l=1}^{N}\left\|u_{\tau}^{l}-u_{\tau}^{l-1}\right\|_{V^{*}}^{2} \leq N \sum_{l=1}^{N}\left\|u_{\tau}^{l}-u_{\tau}^{l-1}\right\|_{V^{*}}^{2} \\
& =T \tau \sum_{l=1}^{N}\left\|\frac{u_{\tau}^{l}-u_{\tau}^{l-1}}{\tau}\right\|_{V^{*}}^{2}=T\left\|u_{\tau}^{\prime}\right\|_{\mathcal{V}^{*}}^{2}
\end{aligned}
$$

This means that (27) holds due to the bound in (26), which completes the proof of the lemma.

Finally, we give the main result of this section.

Theorem 20 Assume that $H(A), H(F), H(\mathscr{N}), H(J), H(M), H(\vartheta), H(0)$ and $H(f)$ hold. Let $\left\{\tau_{n}\right\}$ be a sequence such that $\tau_{n} \rightarrow 0$, as $n \rightarrow \infty$. Then, for a subsequence, still denoted by $\left\{\tau_{n}\right\}$, we have

$$
\begin{aligned}
& \bar{u}_{\tau} \rightarrow u \text { weakly in } \mathcal{V} \text { and } \mathcal{H}, \\
& u_{\tau} \rightarrow u \text { weakly in } \mathcal{V}, \\
& u_{\tau}^{\prime} \rightarrow u^{\prime} \text { weakly in } \mathcal{V}^{*}, \\
& \xi_{\tau} \rightarrow \xi \text { weakly in } \mathcal{X}^{*}, \\
& x_{\tau} \rightarrow x \text { in } C(0, T ; E),
\end{aligned}
$$

where $(x, u, \xi) \in C(0, T ; E) \times \mathcal{W} \times \mathcal{X}^{*}$ is a solution of Problem 14 in the sense of Definition 15.

Proof From the estimates (22)-(24) and the reflexivity of $\mathcal{V}$ and $\mathcal{H}$, without loss of generality, we may assume that there exist $u, \widehat{u} \in \mathcal{V}$ such that convergence (29) holds and $u_{\tau} \rightarrow \widehat{u}$ weakly in $\mathcal{V}$, as $\tau \rightarrow 0$. It is easy to obtain that

$$
\left\|\bar{u}_{\tau}-u_{\tau}\right\|_{\mathcal{V}^{*}}^{2}=\sum_{k=1}^{N} \int_{t_{k-1}}^{t_{k}}\left(t_{k}-s\right)^{2}\left\|\frac{u_{\tau}^{k}-u_{\tau}^{k-1}}{\tau}\right\|_{V^{*}}^{2} d s=\frac{\tau^{2}}{3}\left\|u_{\tau}^{\prime}\right\|_{\mathcal{V}^{*}}^{2} .
$$

This combined with the bound in (26) implies

$$
\bar{u}_{\tau}-u_{\tau} \rightarrow 0_{\mathcal{V}^{*}} \text { in } \mathcal{V}^{*}, \text { as } \tau \rightarrow 0 .
$$


Recalling that $u_{\tau} \rightarrow \widehat{u}$ weakly in $\mathcal{V}$ and using convergence (29), we have $\bar{u}_{\tau}-u_{\tau} \rightarrow u-\widehat{u}$ weakly in $\mathcal{V}$, as $\tau \rightarrow 0$. Moreover, the continuity of embedding $\mathcal{V} \subset \mathcal{V}^{*}$ ensures that $\bar{u}-u_{\tau} \rightarrow u-\widehat{u}$ weakly in $\mathcal{V}^{*}$ as well. So, from (34), we conclude $u=\widehat{u}$, i.e., (30) holds.

The functions $\widehat{u}_{\tau}$ defined in (13) are bounded in $\mathcal{V}$. So, there exists a function $u^{*} \in \mathcal{V}$ such that $\widehat{u}_{\tau} \rightarrow u^{*}$ weakly in $\mathcal{V}$, as $\tau \rightarrow 0$. In the same time, we have

$$
\begin{aligned}
\left\|\widehat{u}_{\tau}-u_{\tau}\right\|_{\mathcal{V}^{*}}^{2} & =\sum_{k=1}^{N} \int_{t_{k-1}}^{t_{k}}\left\|\frac{t-t_{k}+\tau}{\tau}\left(u_{\tau}^{k-1}-u_{\tau}^{k}\right)+\frac{t-t_{k}}{\tau}\left(u_{\tau}^{k-1}-u_{\tau}^{k-2}\right)\right\|_{V^{*}}^{2} d t \\
& \leq 2 \sum_{k=1}^{N} \int_{t_{k-1}}^{t_{k}}\left(t-t_{k-1}\right)^{2}\left\|\frac{u_{\tau}^{k}-u_{\tau}^{k-1}}{\tau}\right\|_{V^{*}}^{2}+\left(t_{k}-t\right)^{2}\left\|\frac{u_{\tau}^{k-1}-u_{\tau}^{k-2}}{\tau}\right\|_{V^{*}}^{2} d t \\
& \leq \frac{2}{3} \tau^{2}\left\|u_{\tau}^{\prime}\right\|_{\mathcal{V}^{*}}^{2} .
\end{aligned}
$$

This implies that $\widehat{u}_{\tau}-u_{\tau} \rightarrow 0_{\mathcal{V}^{*}}$, as $\tau \rightarrow 0$. Similarly, we can conclude that $u^{*}=u$. Moreover, (26) entails that there exists a function $w^{*} \in \mathcal{V}^{*}$ such that

$$
u_{\tau}^{\prime} \rightarrow w^{*} \text { weakly in } \mathcal{V}^{*}, \quad \text { as } \tau \rightarrow 0
$$

This convergence together with (30), by [50, Proposition 23.19] implies that $w^{*}=u^{\prime}$, i.e., convergence (31) is verified. Furthermore, estimate (25) guarantees that there exists a function $\xi \in \mathcal{X}^{*}$ such that convergence (32) holds.

Note that since $u \in \mathcal{V}$, we apply [20, Proposition 5.3, p.66] and [26, Section 4] to conclude that there exists a unique mild solution $x \in C(0, T ; E)$ of the form

$$
x(t)=e^{A(t)} x_{0}+\int_{0}^{t} e^{A(t-s)} f(s, x(s), \vartheta u(s)) d s \quad \text { for a.e. } t \in(0, T),
$$

to problem

$$
\left\{\begin{array}{l}
x^{\prime}(t)=A x(t)+f(t, x(t), \vartheta u(t)) \quad \text { for a.e. } t \in(0, T), \\
x(0)=x_{0}
\end{array}\right.
$$

Now, we return to functions $x_{\tau}$ and $x$, and, for all $t \in[0, T]$, we get

$$
\begin{aligned}
\left\|x_{\tau}(t)-x(t)\right\|_{E} \leq & M_{A} \int_{0}^{t}\left\|f\left(s, x_{\tau}(s), \vartheta \widehat{u}_{\tau}(s)\right)-f(s, x(s), \vartheta u(s))\right\|_{E} d s \\
\leq & M_{A} \int_{0}^{t}\left\|f\left(s, x_{\tau}(s), \vartheta \widehat{u}_{\tau}(s)\right)-f\left(s, x(s), \vartheta \widehat{u}_{\tau}(s)\right)\right\|_{E} d s \\
& \quad+M_{A} \int_{0}^{t}\left\|f\left(s, x(s), \vartheta \widehat{u}_{\tau}(s)\right)-f(s, x(s), \vartheta u(s))\right\|_{E} d s \\
\leq & M_{A} \int_{0}^{t} \varphi(s)\left\|x_{\tau}(s)-x(s)\right\|_{E} d s \\
& +M_{A} \int_{0}^{t}\left\|f\left(s, x(s), \vartheta \widehat{u}_{\tau}(s)\right)-f(s, x(s), \vartheta u(s))\right\|_{E} d s,
\end{aligned}
$$

where $M_{A}:=\max _{t \in[0, T]}\left\|e^{A(t)}\right\|$. We set

$$
h(t)=\int_{0}^{t}\left\|f\left(s, x(s), \vartheta \widehat{u}_{\tau}(s)\right)-f(s, x(s), \vartheta u(s))\right\|_{E} d s
$$


for all $t \in[0, T]$. It follows from Gronwall's inequality and the fact $h(s) \leq h(t)$ for all $s \leq t$ that

$$
\begin{aligned}
\left\|x_{\tau}(t)-x(t)\right\|_{E} & \leq M_{A} h(t)+M_{A}^{2} \int_{0}^{t} h(s) \varphi(s) \exp \left(M_{A} \int_{0}^{r} \varphi(r) d r\right) d s \\
& \leq M_{A} h(t)\left(1+M_{A} \int_{0}^{t} \varphi(s) \exp \left(M_{A} \int_{0}^{r} \varphi(r) d r\right) d s\right) \\
& \leq M_{A} h(t)\left(1+M_{A}\|\varphi\|_{L^{1}} \exp \left(M_{A}\|\varphi\|_{L^{1}}\right)\right)
\end{aligned}
$$

for all $t \in[0, T]$. Since $u_{\tau} \rightarrow u$ weakly in $\mathcal{V}, u_{\tau}^{\prime} \rightarrow u^{\prime}$ weakly in $\mathcal{V}^{*}$, as $\tau \rightarrow 0$, and the embedding $\mathcal{W} \subset C(0, T ; H)$ is continuous, we can see that $u_{\tau} \rightarrow u$ weakly in $C(0, T ; H)$. From [34, Lemma 4], we have

$$
u_{\tau}(t) \rightarrow u(t) \text { weakly in } H, \text { as } \tau \rightarrow 0, \text { for all } t \in[0, T] .
$$

It follows from compactness of $\vartheta$ that

$$
\vartheta\left(\widehat{u}_{\tau}(t)\right) \rightarrow \vartheta(u(t)) \text { in } Y \text {, as } \tau \rightarrow 0, \text { for all } t \in[0, T] .
$$

This combined with (35), by hypothesis $H(f)$ (ii) and the Lebesgue-dominated convergence theorem, see [36, Theorem 1.65], implies

$$
\begin{aligned}
& \lim _{\tau \rightarrow 0}\left\|x_{\tau}-x\right\|_{C(0, T ; E)} \leq m_{1} \lim _{\tau \rightarrow 0} \int_{0}^{T}\left\|f\left(s, x(s), \vartheta \widehat{u}_{\tau}(s)\right)-f(s, x(s), \vartheta u(s))\right\|_{E} d s \\
& \quad \leq m_{1} \int_{0}^{T} \lim _{\tau \rightarrow 0}\left\|f\left(s, x(s), \vartheta \widehat{u}_{\tau}(s)\right)-f(s, x(s), \vartheta u(s))\right\|_{E} d s \rightarrow 0,
\end{aligned}
$$

where $m_{1}:=M_{A}\left(1+M_{A}\|\varphi\|_{L^{1}} \exp \left(M_{A}\|\varphi\|_{L^{1}}\right)\right)$. Hence

$$
x_{\tau} \rightarrow x \text { in } C(0, T ; E), \text { as } \tau \rightarrow 0,
$$

i.e., (33) holds. This convergence together with $H(F)$ gives

$$
\begin{aligned}
& \left\|\frac{1}{\tau} \int_{t_{k-1}}^{t_{k}} F\left(s, x_{\tau}(s)\right) d s-\frac{1}{\tau} \int_{t_{k-1}}^{t_{k}} F(s, x(s)) d s\right\|_{V^{*}} \\
\leq & \frac{1}{\tau} \int_{t_{k-1}}^{t_{k}}\left\|F\left(s, x_{\tau}(s)\right)-F(s, x(s))\right\|_{V^{*}} d s \\
\leq & \max _{s \in[0, T]}\left\|F\left(s, x_{\tau}(s)\right)-F(s, x(s))\right\|_{V^{*}} \rightarrow 0, \text { as } \tau \rightarrow 0 .
\end{aligned}
$$

So, from the Lebesgue-dominated convergence theorem, we have $\mathscr{F}_{\tau}(\cdot)-\overline{\mathscr{F}}_{\tau}(\cdot) \rightarrow 0_{\mathcal{V}^{*}}$ strongly in $\mathcal{V}^{*}$, as $\tau \rightarrow 0$, where $\overline{\mathscr{F}}_{\tau}(t)=\frac{1}{\tau} \int_{t_{k-1}}^{t_{k}} F(s, x(s)) d s$ for $t \in\left[t_{k-1}, t_{k}\right), k=$ $1,2, \ldots, N$. Exploiting the fact that $x \in C(0, T ; E)$, by hypothesis $H(f)$ and $[6$, Lemma 3.3], we have

$$
\mathscr{F}_{\tau}(\cdot) \rightarrow \mathscr{F}(\cdot):=F(\cdot, x(\cdot)) \quad \text { in } \mathcal{V}^{*}, \quad \text { as } \tau \rightarrow 0 .
$$

It remains to verify that $(x, u, \xi)$ is a mild solution to Problem 14. The convergence (31) guarantees that

$$
\left(u_{\tau}^{\prime}, v\right)_{\mathcal{H}}=\left\langle u_{\tau}^{\prime}, v\right\rangle_{\mathcal{V}^{*} \times \mathcal{V}} \rightarrow\left\langle u^{\prime}, v\right\rangle_{\mathcal{V}^{*} \times \mathcal{V}}=\left(u^{\prime}, v\right)_{\mathcal{H}}
$$

for all $v \in \mathcal{V}$. Next, for the Nemytskii operator $\widetilde{N}$, we observe that if $H(\mathscr{N})(\text { ii })_{1}$ occurs, then it follows from the uniform bound of $\left\{\bar{u}_{\tau}\right\} \subset M^{2,2}\left(0, T ; V, V^{*}\right)$, see (27), the convergence $\bar{u}_{\tau} \rightarrow u$ weakly in $\mathcal{V}$, as $\tau \rightarrow 0$, and [17, Lemma 1] that

$$
\widetilde{\mathscr{N}} u_{\tau} \rightarrow \widetilde{\mathscr{N}} u \text { weakly in } \mathcal{V}^{*} \text {, as } \tau \rightarrow 0 \text {. }
$$


Obviously, the above convergence holds also, when hypothesis $H(\mathscr{N})(\mathrm{ii})_{2}$ is satisfied, since $u_{\tau} \rightarrow u$ weakly in $\mathcal{V}$, as $\tau \rightarrow 0$. Therefore, we conclude

$$
\left\langle\widetilde{\mathscr{N}} \bar{u}_{\tau}, v\right\rangle_{\mathcal{V}^{*} \times \mathcal{V}} \rightarrow\langle\widetilde{\mathscr{N}} u, v\rangle_{\mathcal{V}^{*} \times \mathcal{V}}
$$

for all $v \in \mathcal{V}$. The convergence (32) implies

$$
\left\langle\xi_{\tau}, \mathcal{M} v\right\rangle_{\mathcal{X}^{*} \times \mathcal{X}} \rightarrow\langle\xi, \mathcal{M} v\rangle_{\mathcal{X}^{*} \times \mathcal{X}}
$$

for all $v \in \mathcal{V}$. Furthermore, from (36), we have

$$
\left\langle\mathscr{F}_{\tau}, v\right\rangle_{\mathcal{V} \times \mathcal{V}}=\langle\mathscr{F}, v\rangle_{\mathcal{V}^{\times} \mathcal{V}}
$$

for all $v \in \mathcal{V}$. Combining with (37)-(40), we obtain

$$
\left(u^{\prime}, v\right)_{\mathcal{H}}+\langle\widetilde{\mathscr{N}} u, v\rangle+\langle\xi, \mathcal{M} v\rangle_{\mathcal{X}} \times \mathcal{X}=\langle F, v\rangle_{\mathcal{V} \times \mathcal{V}}
$$

for all $v \in \mathcal{V}$.

To complete the proof of the theorem, we need to prove that $\xi(t) \in \partial J(x(t), M u(t))$ for a.e. $t \in(0, T)$. From (27), (29) and hypothesis $H(M)$, we have

$$
\mathcal{M}\left(\bar{u}_{\tau}\right) \rightarrow \mathcal{M}(u) \text { in } \mathcal{X}^{*}, \text { as } \tau \rightarrow 0 .
$$

So, we may suppose, passing to a subsequence if necessary, that

$$
M \bar{u}_{\tau}(t) \rightarrow M u(t) \text { in } X^{*} \text {, for a.e. } t \in(0, T) .
$$

On the other hand, (33) ensures that $x_{\tau}(t) \rightarrow x(t)$ in $E$ for all $t \in[0, T]$. Furthermore, since $\xi_{\tau} \rightarrow \xi$ weakly in $\mathcal{X}^{*}$ and $\partial J$ has weakly compact and convex values, we use Lemma 13 and the Aubin-Cellina convergence theorem, see [2, Theorem 1, p.60], to conclude

$$
\xi(t) \in \partial J(x(t), M u(t)) \text { for a.e. } t \in(0, T) .
$$

Consequently, we have shown that the triple of functions $(x, u, \xi) \in C(0, T ; E) \times \mathcal{W} \times \mathcal{X}^{*}$ is a mild solution to Problem 14 in the sense of Definition 15. This completes the proof of the theorem.

Open Access This article is distributed under the terms of the Creative Commons Attribution 4.0 International License (http://creativecommons.org/licenses/by/4.0/), which permits unrestricted use, distribution, and reproduction in any medium, provided you give appropriate credit to the original author(s) and the source, provide a link to the Creative Commons license, and indicate if changes were made.

\section{References}

1. Ahmed, N.U.: Optimal control of hydrodynamic flow with possible application to artificial heart. Dyn. Syst. Appl. 1, 103-120 (1992)

2. Aubin, J.P., Cellina, A.: Differential Inclusions: Set-Valued Maps and Viability Theory. Springer, Berlin (1984)

3. Bartosz, K.: Numerical methods for evolution hemivariational inequalities, Chapter 5. In: Han, W., et al. (eds.) Advances in Variational and Hemivariational Inequalities with Applications. Theory, Numerical Analysis, and Applications. Advances in Mechanics and Mathematics, vol. 33, pp. 111-144. Springer, Berlin (2015)

4. Bartosz, K., Cheng, X.L., Kalita, P., Yu, Y.J., Zheng, C.: Rothe method for parabolic variationalhemivariational inequalities. J. Math. Anal. Appl. 423, 841-862 (2015)

5. Bartosz, K., Sofonea, M.: The Rothe method for variational-hemivariational inequalities with applications to contact mechanics. SIAM. J. Math. Anal. 48, 886-883 (2016) 
6. Carstensen, C., Gwinner, J.: A theory of discretization for nonlinear evolution inequalities applied to parabolic Signorini problems. Ann. Mat. Pura Appl. 177, 363-394 (1999)

7. Chen, X., Wang, Z.: Convergence of regularized time-stepping methods for differential variational inequalities. SIAM J. Optim. 23, 1647-1671 (2013)

8. Chen, X., Wang, Z.: Differential variational inequality approach to dynamic games with shared constraints. Math. Program. 146, 379-408 (2014)

9. Denkowski, Z., Migórski, S., Papageorgiou, N.S.: An Introduction to Nonlinear Analysis: Theory. Kluwer Academic, Boston (2003)

10. Denkowski, Z., Migórski, S., Papageorgiou, N.S.: An Introduction to Nonlinear Analysis: Applications. Kluwer Academic, Boston (2003)

11. Fang, C.J., Han, W.M., Migórski, S., Sofonea, M.: A class of hemivariational inequalities for nonstationary Navier-Stokes equations. Nonlinear Anal. Real World Appl. 31, 257-276 (2016)

12. Gwinner, J.: On a new class of differential variational inequalities and a stability result. Math. Program. 139, 205-221 (2013)

13. Han, L., Pang, J.S.: Non-zenoness of a class of differential quasi-variational inequalities. Math. Program. 121, 171-199 (2010)

14. Han, W., Migórski, S., Sofonea, M. (eds.): Advances in Variational and Hemivariational Inequalities with Applications.Theory, Numerical Analysis, and Applications. Advances in Mechanics and Mathematics, vol. 33. Springer, Berlin (2015)

15. Han, W., Sofonea, M.: Quasistatic Contact Problems in Viscoelasticity and Viscoplasticity. Studies in Advanced Mathematics, vol. 30. Americal Mathematical Society, Providence (2002)

16. Kačur, J.: Method of Rothe in Evolution Equations, Teubner-Texte zur Mathematik, vol. 80. B.G. Teubner, Leipzig (1985)

17. Kalita, P.: Convergence of Rothe scheme for hemivariational inequalities of parabolic type. Int. J. Numer. Anal. Model. 10, 445-465 (2013)

18. Li, X.S., Huang, N.J., O'Regan, D.: Differential mixed variational inequalities in finite dimensional spaces. Nonlinear Anal. Theory Methods Appl. 72, 3875-3886 (2010)

19. Li, X.S., Huang, N.J., O'Regan, D.: A class of impulsive differential variational inequalities in finite dimensional spaces. J. Franklin Inst. 353, 3151-3175 (2016)

20. Li, X.J., Yong, J.M.: Optimal control theory for infinite dimensional systems. Springer, Boston (2012)

21. Liu, Z.H.: Existence results for quasilinear parabolic hemivariational inequalities. J. Differ. Equ. 244, 1395-1409 (2008)

22. Liu, Z.H., Loi, N.V., Obukhovskii, V.: Existence and global bifurcation of periodic solutions to a class of differential variational inequalities. Int. J. Bifurcat. Chaos 23, 1350125 (2013)

23. Liu, Z.H., Migórski, S., Zeng, S.D.: Partial differential variational inequalities involving nonlocal boundary conditions in Banach spaces. J. Differ. Equ. 263, 3989-4006 (2017)

24. Liu, Z.H., Motreanu, D., Zeng, S.D.: On the well-posedness of differential mixed quasi-variational inequalities. Topol. Method Nonlinear Anal. 51, 135-150 (2019)

25. Liu, Z.H., Zeng, S.D.: Differential variational inequalities in infinite Banach spaces. Acta Math. Sci. 37, 26-32 (2017)

26. Liu, Z.H., Zeng, S.D., Motreanu, D.: Evolutionary problems driven by variational inequalities. J. Differ. Equ. 260, 6787-6799 (2016)

27. Liu, Z.H., Zeng, S.D., Motreanu, D.: Partial differential hemivariational inequalities. Adv. Nonlinear Anal. (2017). https://doi.org/10.1515/anona-2016-0102

28. Liu, Z.H., Zeng, S.D., Zeng, B.: Well-posedness for mixed quasi-variational-hemivariational inequalities. Topol. Methods Nonlinear Anal. 47, 561-578 (2016)

29. Loi, N.V.: On two-parameter global bifurcation of periodic solutions to a class of differential variational inequalities. Nonlinear Anal. Theory Methods Appl. 122, 83-99 (2015)

30. Migórski, S., Zeng, S.D.: Hyperbolic hemivariational inequalities controlled by evolution equations with application to adhesive contact model. Nonlinear Anal. Real World Appl. 43, 121-143 (2018)

31. Migórski, S., Ochal, A.: Optimal control of parabolic hemivariational inequalities. J. Glob. Optim. 17, 285-300 (2000)

32. Migórski, S., Ochal, A.: Hemivariational inequalities for stationary Navier-Stokes equations. J. Math. Anal. Appl. 306, 197-217 (2005)

33. Migórski, S., Ochal, A.: Navier-Stokes problems modeled by evolution hemivariational inequalities. Discret. Contin. Dyn. Syst. Suppl. 2007, 731-740 (2007)

34. Migórski, S., Ochal, A.: Quasi-static hemivariational inequality via vanishing acceleration approach. SIAM J. Math. Anal. 41, 1415-1435 (2009)

35. Migórski, S., Ochal, A., Sofonea, M.: History-dependent subdifferential inclusions and hemivariational inequalities in contact mechanics. Nonlinear Anal. Real World Appl. 12, 3384-3396 (2011) 
36. Migórski, S., Ochal, A., Sofonea, M.: Nonlinear Inclusions and Hemivariational Inequalities. Models and Analysis of Contact Problems. Advances in Mechanics and Mathematics, vol. 26. Springer, New York (2013)

37. Panagiotopoulos, P.D.: Inequality Problems in Mechanics and Applications. Birkhäuser, Boston (1985)

38. Panagiotopoulos, P.D.: Hemivariational Inequalities, Applications in Mechanics and Engineering. Springer, Berlin (1993)

39. Pang, J.S.: Frictional contact models with local compliance: semismooth formulation. J. Appl. Math. Mech. (ZAMM Z. Angew. Math. Mech.) 88, 454-471 (2008)

40. Pang, J.S., Han, L., Ramadurai, G., Ukkusuri, S.: A continuous-time linear complementarity system for dynamic user equilibria in single bottleneck traffic flows. Math. Program. 133, 437-460 (2012)

41. Pang, J.S., Stewart, D.E.: Differential variational inequalities. Math. Program. 113, 345-424 (2008)

42. Papageorgiou, N.S., Rădulescu, V.D., Repov̌̌, D.D.: Nonlinear elliptic inclusions with unilateral constraint and dependence on the gradient. Appl. Math. Optim. (2016). https://doi.org/10.1007/s00245-0169392-y

43. Papageorgiou, N.S., Rădulescu, V.D., Repovš, D.D.: Nonhomogeneous hemivariational inequalities with indefinite potential and Robin boundary condition. J. Optim. Theory Appl. (2017). https://doi.org/10. 1007/s10957-017-1173-5

44. Shillor, M., Sofonea, M., Telega, J.J.: Models and Analysis of Quasistatic Contact, vol. 655. Springer, Berlin (2004). Lecture Notes Physics

45. Sofonea, M., Han, W.M., Shillor, M.: Analysis and Approximation of Contact Problems with Adhesion or Damage. Chapman \& Hall, Boca Raton (2006)

46. Stewart, D.E.: Uniqueness for solutions of differential complementarity problems. Math. Program. 118, 327-345 (2009)

47. Van, N.T., Ke, T.D.: Asymptotic behavior of solutions to a class of differential variational inequalities. Ann. Pol. Math. 114, 147-164 (2015)

48. Wang, X., Huang, N.J.: A class of differential vector variational inequalities in finite dimensional spaces. J. Optim. Theory Appl. 162, 633-648 (2014)

49. Xiao, Y.B., Huang, N.J.: Browder-Tikhonov regularization for a class of evolution second order hemivariational inequalities. J. Glob. Optim. 45, 371-388 (2009)

50. Zeidler, E.: Nonlinear Functional Analysis and Applications II A/B. Springer, New York (1990)

51. Zeng, S.D., Migórski, S.: Noncoercive hyperbolic variational inequalities with applications to contact mechanics. J. Math. Anal. Appl. 455, 619-637 (2017)

52. Zeng, S.D., Migórski, S.: A Class of time-fractional hemivariational inequalities with application to frictional contact problem. Commun. Nonlinear Sci. Numer. Simul. 56, 34-48 (2018)

53. Zeng, S.D., Liu, Z.H., Migórski, S.: A class of fractional differential hemivariational inequalities with application to contact problem. Z. Angew. Math. Phys 69, 36 (2018). pages 23 